\title{
Carbon Dioxide Laser Treatment of Cutaneous Neurofibromas
}

Yi Zhen Chiang $\cdot$ Firas Al-Niaimi $\cdot$ Janice Ferguson $\cdot$ Paul Jeffrey August $\cdot$ Vishal Madan

To view enhanced content go to www.dermtherapy-open.com

Received: December 6, 2011 / Published online: May 25, 2012

(c) The Author(s) 2012. This article is published with open access at Springerlink.com

\section{ABSTRACT}

Introduction: Neurofibromatosis type 1 (NF1) is an autosomal dominant disorder, with multisystem involvement, including cutaneous manifestations of hyperpigmentation and neurofibromas. Multiple cutaneous lesions are often disfiguring and lead to emotional distress and social isolation. Treatment of NF1 is predominantly surgical but alternative treatments should be considered for patients with large numbers of lesions as cold steel excision of multiple lesions can be cumbersome

This study was presented at the British Society of Dermatological Surgery section of the 90th meeting of the British Association of Dermatologists' in London, July 2011.

Y. Z. Chiang · F. Al-Niaimi · J. Ferguson ·

P. J. August · V. Madan ( $\square)$

The Dermatology Centre, Salford Royal NHS Foundation Trust, Manchester, UK e-mail: vishal.madan@srft.nhs.uk

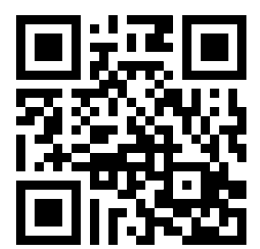

Enhanced content for this article is available on the journal web site: www.dermtherapy-open.com and may not be practical. The authors report a series of patients with multiple neurofibromas successfully treated using a $\mathrm{CO}_{2}$ laser.

Methods: Data on $\mathrm{CO}_{2}$ laser treatments, followup, and recurrence following treatment was collected retrospectively. A post-treatment telephone survey was carried out to assess patient satisfaction using a standardized set of questions and a scoring tool.

Results: Five of seven patients who underwent $\mathrm{CO}_{2}$ laser treatment of their multiple neurofibromas responded to the posttreatment survey. All five patients (age range 36-56 years, mean age 45.2 years, three men:two women) had multiple variable-sized neurofibromas. The mean number of lesions per patient was 114 (range 20-200 lesions). The mean number of treatment sessions was 2.2 (range 1-4 sessions) and mean follow-up was 14.4 months (range 6-24 months). Three patients $(60 \%)$ reported no recurrence up to 2 years post-laser treatment. Two patients (40\%) had recurrences of a few lesions $(\leq 10 \%$ of treated lesions per patient). The mean patient satisfaction score was 9.2 out of 10 (range 8-10). All patients mentioned that they would recommend $\mathrm{CO}_{2}$ laser treatment to others with 
multiple neurofibromas. Hypopigmentation or depigmentation at treatment sites were the only reported adverse effects.

Conclusion: Based on current results, the authors feel that $\mathrm{CO}_{2}$ laser treatment achieves a high level of patient satisfaction with a low recurrence of treated lesions.

Keywords: Carbon dioxide laser; Laser; Neurofibroma; Neurofibromatosis; Patient satisfaction

\section{INTRODUCTION}

Neurofibromatosis type 1 (NF1), formerly known as Von Recklinghausen's disease, is an autosomal dominant inherited condition with an incidence of 1:2,500-1:3,000, and a prevalence of 1:4,000-1:5,000 [1]. Clinical manifestations of NF1 involve multiple systems, including the nervous system, bone, and skin, with patients developing manifestations of hyperpigmentation and neurofibromas [2]. The National Institute of Health (NIH) Consensus Development Conference have determined the diagnostic criteria for NF1, which requires two or more of the following: six or more café au lait macules; two or more cutaneous/subcutaneous neurofibromas, or one plexiform neurofibroma; axillary/groin freckling; optic glioma; two or more Lisch nodules in the eyes; bony dysplasia; and a first-degree relative with NF1 [3].

Cutaneous neurofibromas can affect any part of the body and can vary in size, number, and distribution. Neurofibromas usually appear around puberty and continue to grow in size and number [4]. Significant disfigurement can result from the growth of hundreds of cutaneous neurofibromas, leading to social isolation and emotional distress [5].
Treatment of cutaneous neurofibromas is predominantly surgical but alternative treatments need to be considered for patients with multiple lesions often in excess of 100, in whom surgical intervention may not be possible or desirable [6]. The $\mathrm{CO}_{2}$ laser has been shown to be effective in treating large numbers of small-to-medium sized neurofibromas with cosmetic outcomes equal to or even better than surgical excision [2, 7]. However, evidence for $\mathrm{CO}_{2}$ effectiveness, effect of treatment on patient satisfaction, and rate of recurrence post-treatment is scarce. The authors evaluated a series of patients with multiple neurofibromas that were successfully treated using $\mathrm{CO}_{2}$ laser treatment under general anesthetic, and assessed patient satisfaction with the treatment and rate of recurrence post-treatment.

\section{MATERIALS AND METHODS}

\section{Patients}

The authors' inclusion criteria included patients who were diagnosed with NF1, fulfilled the NIH Consensus Development Conference NF1 diagnostic criteria, had more than 100 neurofibromas of varying sizes, and were treated with $\mathrm{CO}_{2}$ laser treatment over the last 4 years. Seven NF1 patients satisfied these inclusion criteria and were included. Informed consent was obtained from five patients (71\% response rate) for a post-treatment telephone survey.

\section{Carbon Dioxide Laser Treatment Procedure}

All patients underwent test patches with the $\mathrm{CO}_{2}$ laser and were reviewed at 3-6 months 
when the results were assessed. If satisfactory, patients were then invited for $\mathrm{CO}_{2}$ laser treatment under general anesthetic.

The Sharplan ${ }^{\circledR} 40$ C Silktouch laser (Laser Industries Ltd., Tel Aviv, Israel) was used in its freehand continuous mode $(10-20 \mathrm{~W}$ with rapid side-to-side hand movements) or in scanner settings $(125 \mathrm{~mm}$ hand piece, $7-14 \mathrm{~W}, 3 \mathrm{~mm}$ spot) to ablate the small neurofibromas. Peripheries of larger lesions were first scored with the continuous laser beam. The incision was then extended as the neurofibroma was held with forceps and the entire dumbbell-shaped lesion was excised using the laser. Bleeding was rarely encountered and was easily controlled using the defocused laser beam or Vicryl ${ }^{\circledR}$ (Johnson \& Johnson, NJ, USA).

Postoperatively, antibacterial ointment (mupirocin, Bactroban ${ }^{\circledR}$; Smithkline Beecham Corporation, Philadelphia, PA, USA) was applied under hydrocolloid dressings as the laser wounds healed with secondary intention. Wounds that were considered large enough for delayed secondary intention healing were sutured with Vicryl. All patients were followed up 3 and 6 months later to review the wound, scarring, and cosmetic outcome of treated neurofibroma lesions.

\section{Data Collection}

Five patients completed the post-treatment telephone survey, which was conducted by the same doctor. Patients were asked to rate their satisfaction of $\mathrm{CO}_{2}$ laser treatment (score 0-10) and whether they would recommend $\mathrm{CO}_{2}$ laser treatment to other patients. Retrospective data on $\mathrm{CO}_{2}$ laser treatments, follow-up, and recurrence following treatment was obtained.

\section{RESULTS}

\section{Clinical Outcomes}

All patients had more than 100 neurofibromas of varying sizes. The mean age of patients was 45.2 (age range 36-56) with a male/female ratio of 3:2. Four patients had truncal neurofibromas and one had treatment for neurofibromas on facial, neck, and upper limb skin. Patients received a mean of 2.2 treatment sessions (range $1-4$ ). The mean number of lesions per patient was 114 (range 20-200 lesions). The mean follow-up period was 14.4 months (range 6-24 months). Three patients (60\%) had no lesional recurrence 2 years post- $\mathrm{CO}_{2}$ laser treatment. Two patients (40\%) had recurrence of a few of the treated lesions ( $\leq 10 \%$ of treated lesions per patient). Preoperative and postoperative photos are shown in Figs. 1, 2 and 3. Healing took 3-4 weeks. The wounds were erythematous for approximately 3-6 months, and matured into hypopigmented and sometimes atrophic scars. There were no significant complications. All five patients reported that treatment-induced scarring was cosmetically preferable to neurofibroma lesions. Hypopigmentation or depigmentation at treatment sites were the only reported adverse effects.

\section{Patient Satisfaction}

The mean patient satisfaction score was 9.2 out of 10 (range 8-10). All five patients (100\%) commented that they would recommend $\mathrm{CO}_{2}$ laser treatment to other patients with neurofibromas.

\section{DISCUSSION}

Surgical excision is the standard procedure for removal of lesions, with advantages of a neat 


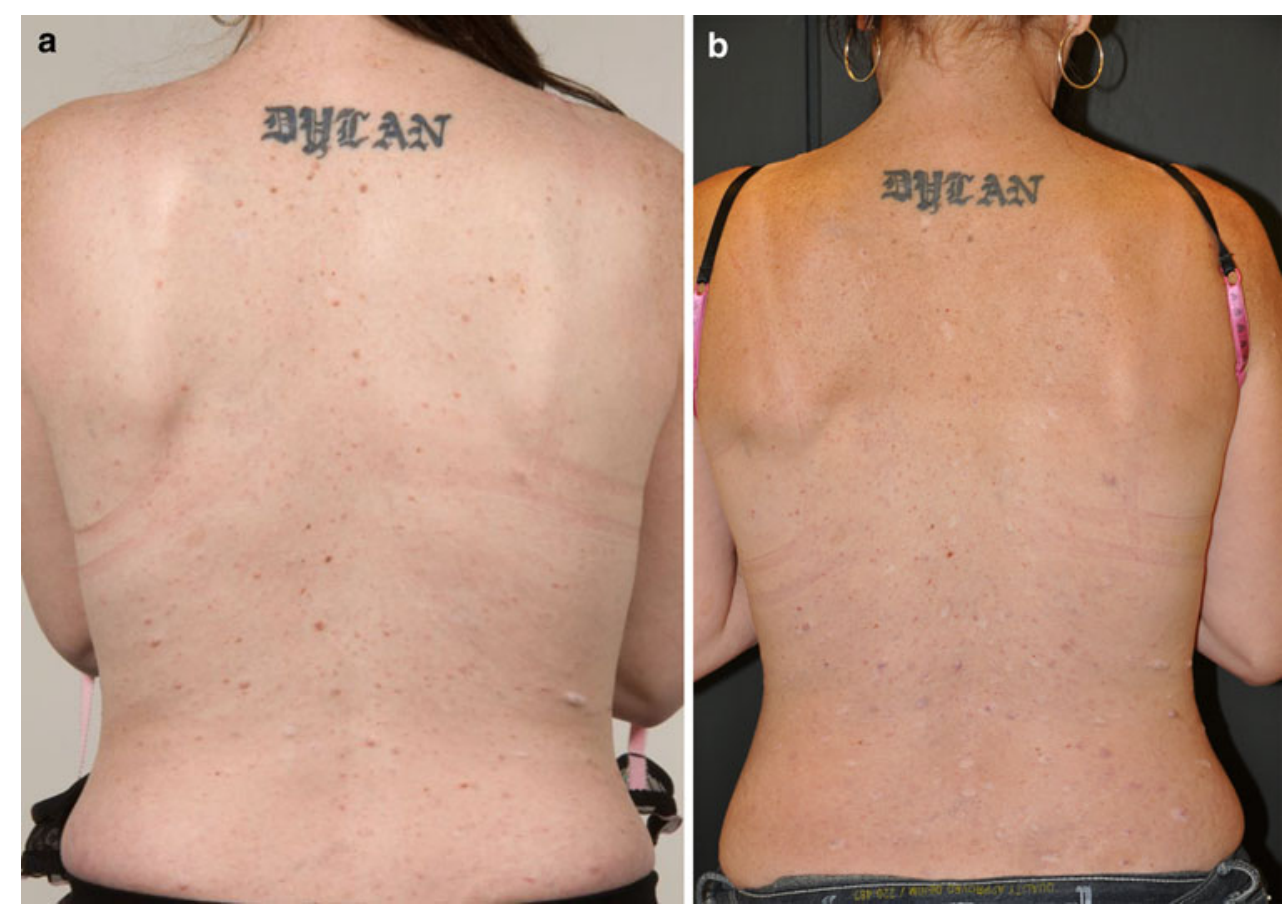

Fig. 1 Patient 1. Before (a) and 6 months after (b) excision of neurofibromas with the $\mathrm{CO}_{2}$ laser

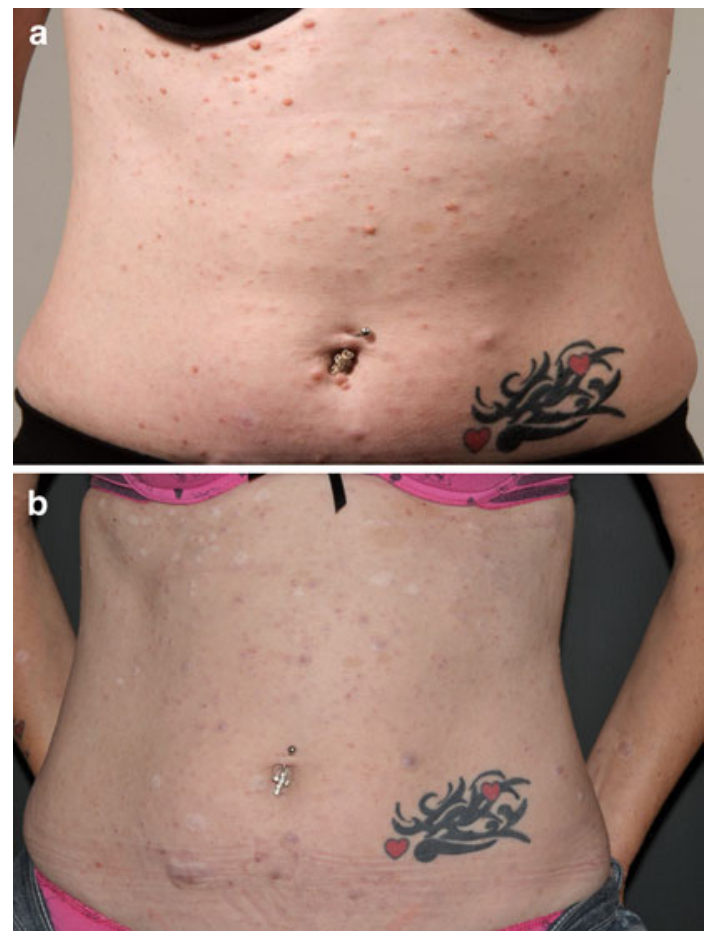

Fig. 2 Patient 2. Before (a) and 6 months after (b) excision of neurofibromas with the $\mathrm{CO}_{2}$ laser linear scar and a specimen for histology. The disadvantages are a longer procedure time and higher risk of bleeding [6]. $\mathrm{CO}_{2}$ laser treatment is suitable for removing large numbers of neurofibromas in a short time with good cosmetic outcomes and a low complication rate $[2,8]$. The current study confirmed that $\mathrm{CO}_{2}$ laser treatment under general anesthetic for hundreds of small-to-medium-sized cutaneous neurofibromas has a high patient satisfaction and recommendation rate.

Previous studies have demonstrated that $\mathrm{CO}_{2}$ laser treatment improved patients' self-confidence, social, and sexual lives [2, 8]. Algermissen et al. [2] reported that the depigmented scars from laser treatment were more acceptable to patients than scars from surgical excision, a finding was echoed by patients in the current study. Previously reported physical and psychological benefits following $\mathrm{CO}_{2}$ laser are summarized in Table $1[2,8]$. 

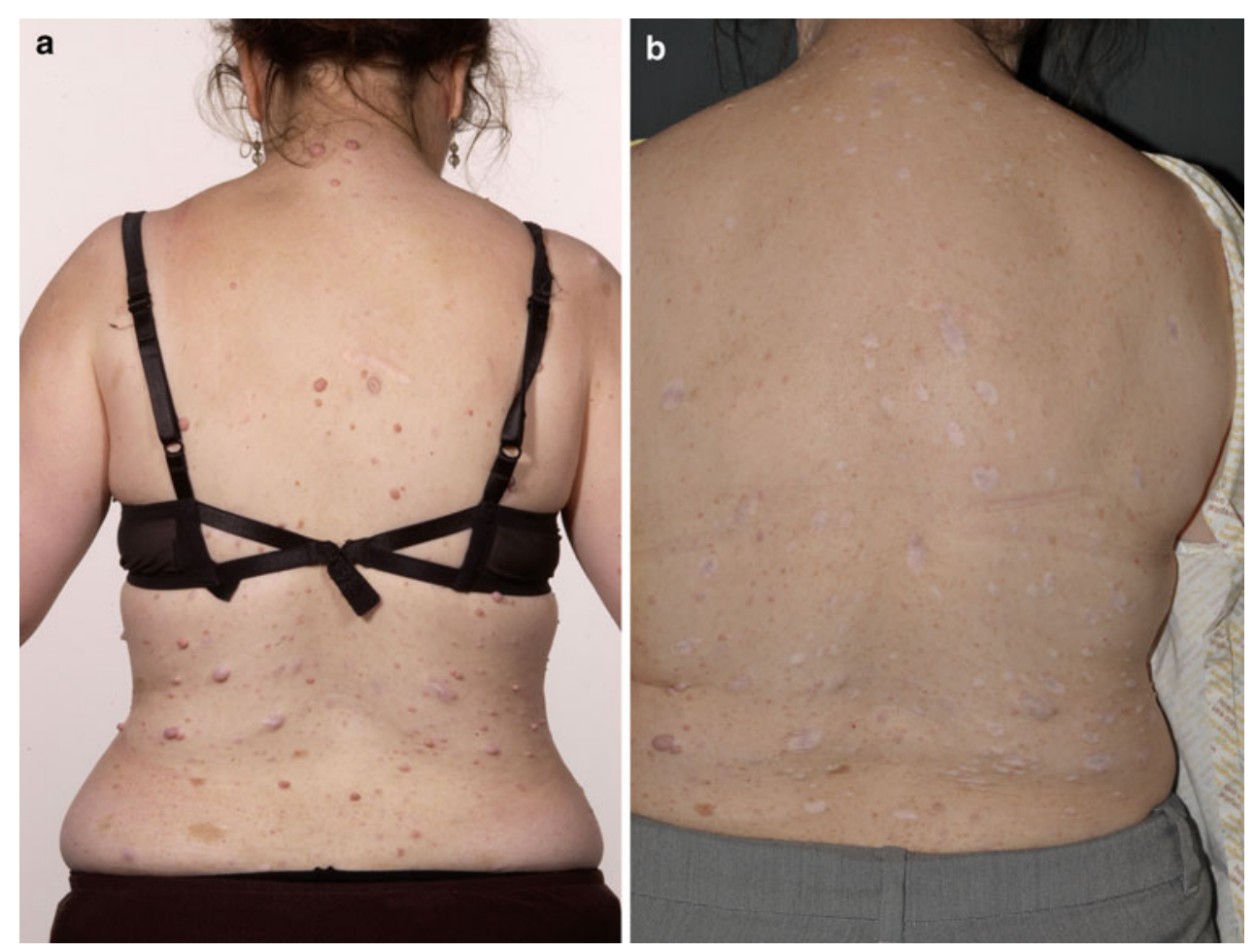

Fig. 3 Patient 3. Before (a) and 6 months after (b) excision of neurofibromas with the $\mathrm{CO}_{2}$ laser

Table 1 Comparison of results from the current study with the published literature

\begin{tabular}{ll}
\hline Studies & Main findings \\
\hline Moreno et al. [8] & $82 \%$ reported good level of improvement in symptoms (e.g., pain, pruritus), and social activity \\
& $73 \%$ reported good level of improvement in sexual activity \\
& $100 \%$ reported good level of improvement from feeling of despair \\
& $73 \%$ satisfaction and recommendation rate \\
& Most reported an increase in self-confidence and acceptance from the social surrounding \\
Algermissen et al. [2] & 9.2 mean satisfaction score (score out of 10) \\
& $100 \%$ recommendation rate \\
\hline
\end{tabular}

Patients in the current study reported high satisfaction levels, suggesting that $\mathrm{CO}_{2}$ laser treatment can potentially improve patients' psychological well-being.

To the authors' knowledge, there have been no previous reports of recurrences following $\mathrm{CO}_{2}$ laser treatment. The current study showed that $40 \%$ of patients had regrowth of some lesions within 2 years, but these represented $\leq 10 \%$ of treated lesions per patient.

The current study, however, is limited by the small number of patients. Patient skin type and skin color was not documented at the start of treatment and, hence, the authors were unable to assess the potential relationship between posttreatment pigmentary changes and skin color. 
There may also be a potential bias with the use of a telephone survey in assessing patient satisfaction levels. In addition, the short followup post-treatment in the current study may not allow the authors to fully assess the recurrence of neurofibroma lesions.

Future studies with a larger number of patients, longer follow-up period, and studies that offer comparative data of $\mathrm{CO}_{2}$ laser treatment with surgical treatment of cutaneous neurofibromas are needed for further clarification of the effectiveness of $\mathrm{CO}_{2}$ laser treatment for cutaneous neurofibromas.

\section{CONCLUSION}

The authors found that $\mathrm{CO}_{2}$ laser treatment for cutaneous neurofibromas is associated with a low recurrence rate, achieves a high level of patient satisfaction, and potentially offers a useful alternative to surgical excision, particularly in patients with multiple lesions.

\section{ACKNOWLEDGMENTS}

The authors would like to thank the staff at the Dermatology Centre, Salford Royal NHS Foundation Trust, Manchester, for their cooperation during this study. Dr. Madan is the guarantor for this article, and takes responsibility for the integrity of the work as a whole.

Conflict of interest. The authors declare no conflict of interest.
Open Access. This article is distributed under the terms of the Creative Commons Attribution Noncommercial License which permits any noncommercial use, distribution, and reproduction in any medium, provided the original author(s) and source are credited.

\section{REFERENCES}

1. Ferner RE. Neurofibromatosis 1 and neurofibromatosis 2: a twenty first century perspective. Lancet Neurol. 2007;6:340-51.

2. Algermissen B, Müller U, Katalinic D, Berlien HP. $\mathrm{CO}_{2}$ laser treatment of neurofibromas of patients with neurofibromatosis type 1: five years experience. Med Laser Appl. 2001;16:265-74.

3. National Institutes of Health Consensus Development Conference. Conference statement. Neurofibromatosis. Arch Neurol. 1988;45:575-8.

4. Boyd KP, Korf BR, Theos A. Neurofibromatosis type 1 . J Am Acad Dermatol. 2009;61:1-14.

5. Mouridsen SE, Sorensen SA. Psychological aspects of von Recklinghausen neurofibromatosis (NF1). J Med Genet. 1995;32:921-4.

6. Becker DW. Use of the carbon dioxide laser in treating multiple cutaneous neurofibromas. Ann Plast Surg. 1991;26:582-6.

7. Boenigk RK, Ratz JL. $\mathrm{CO}_{2}$ laser treatment of cutaneous neurofibromas. J Dermatol Surg Oncol. 1987;13: 187-90.

8. Moreno JC, Mathoret C, Lantieri L, et al. Carbon dioxide laser for removal of multiple cutaneous neurofibromas. Br J Dermatol. 2001;144:1096-8. 